\title{
Endoscopic management of hydrocephalus secondary to tumors of the posterior third ventricle
}

\section{Charles Teo, M.B.B.S., F.R.A.C.S., and Ronald Young II, M.D.}

Centre for Minimally Invasive Neurosurgery, Prince of Wales Hospital/University of New South Wales, Sydney, Australia; and Department of Neurosurgery, University of Texas, Houston, Texas

Management of the obstructive hydrocephalus that accompanies tumors located in the third ventricle has traditionally involved either urgent tumor resection, with resultant ventricular decompression, or cerebrospinal fluid diversion that requires either ventriculostomy or shunt placement prior to tumor removal. Although this approach has worked well for the better part of a century, it has both short- and long-term sequelae that can possibly be avoided. Beacause a number of lesions in this area are benign or are amenable to radiotherapy, a less invasive approach to their treatment is desirable. The advances in both instrumentation and techniques of endoscopic surgery have established alternatives to the traditional treatment of third ventricular tumors and resultant hydrocephalus. The authors review the treatment of 19 patients with posterior third ventricular tumors who presented to Arkansas Children's Hospital over a 5-year period (September 1993-July 1999). In 11 patients signs and/or symptoms of hydrocephalus were demonstrated and were treated with endoscopic third ventriculostomy, additionally, a biopsy procedure, resection, or fenestration of the tumor was performed in a number of patients. Endoscopy was believed to have been of benefit in all patients, despite the eventual failure of the ventriculostomy in one patient. There were no complications in this series. The algorithm thus developed by the authors provides both a diagnostic and therapeutic pathway that may ultimately reduce the morbidity associated with the treatment of patients with posterior third ventricular lesions.

\section{Key Words * endoscopy * hydrocephalus * pineal tumor * third ventricle * brain tumor}

The management of hydrocephalus caused by tumors of the posterior third ventricle is controversial.[8] Indeed, the need for any type of cerebrospinal fluid (CSF) diversion is debatable. However, once the decision to treat the hydrocephalus is made, options include external ventricular drainage, placement of a permanent ventriculoperitoneal shunt, internal CSF diversion, either endoscopic (endoscopic third ventriculostomy [ETV]) or ventriculocisternal shunting (Torkildsen's procedure), and urgent tumor resection in which CSF is not diverted. The advantages of endoscopic management of hydrocephalus over more mainstream techniques such as external ventricular drainage and placement of a ventriculoperitoneal shunt, are poorly defined and certainly not established. Complication rates have been shown to be unacceptably high (up to 75\%) in the recent literature.[2] Our aim in this series is to 
define the role of endoscopy in the management of hydrocephalus secondary to posterior third ventricular tumors, with particular emphasis on issues of safety and reliability.

\section{CLINICAL MATERIAL AND METHODS}

We have used endoscopy extensively in the management of simple and complex hydrocephalus of all types.[8-10] All patients who presented to the Arkansas Children's Hospital from September 1993 to July 1999 with suspected noncommunicating hydrocephalus were given the option of undergoing ETV. If patients, of any age, had acquired noncommunicating hydrocephalus, they were strongly encouraged to undergo this procedure. If they had congenital hydrocephalus and were younger than the age of 2 years, however, we strongly discouraged this treatment. Patients who harbored tumors of the posterior third ventricle and in whom acute, life-threatening secondary hydrocephalus was demonstrated constituted a small percentage of the overall patients with CSF circulation disorders. Indeed, if in those patients with ventriculomegaly signs or symptoms of intracranial hypertension were not shown or if they had relatively longstanding hydrocephalus and associated mild and chronic symptoms of raised intracranial pressure, they were not offered any acute treatment for their hydrocephalus. Rather, treatment was aimed at the primary tumor in the hope that the hydrocephalus would resolve with tumor reduction. If ETV was performed, a sample of CSF was obtained for tumor markers, and a biopsy sample of the tumor was acquired, the tumor was removed, or in the case of cystic lesions, it was drained at the time of the ventriculostomy. All patients underwent some form of diagnostic imaging before surgery. Magnetic resonance imaging was reserved for those patients who did not present with life-threatening hydrocephalus. Those patients who presented with rapidly deteriorating clinical status were taken to surgery after a computerized tomography scan alone had been obtained (Fig. 1 left).

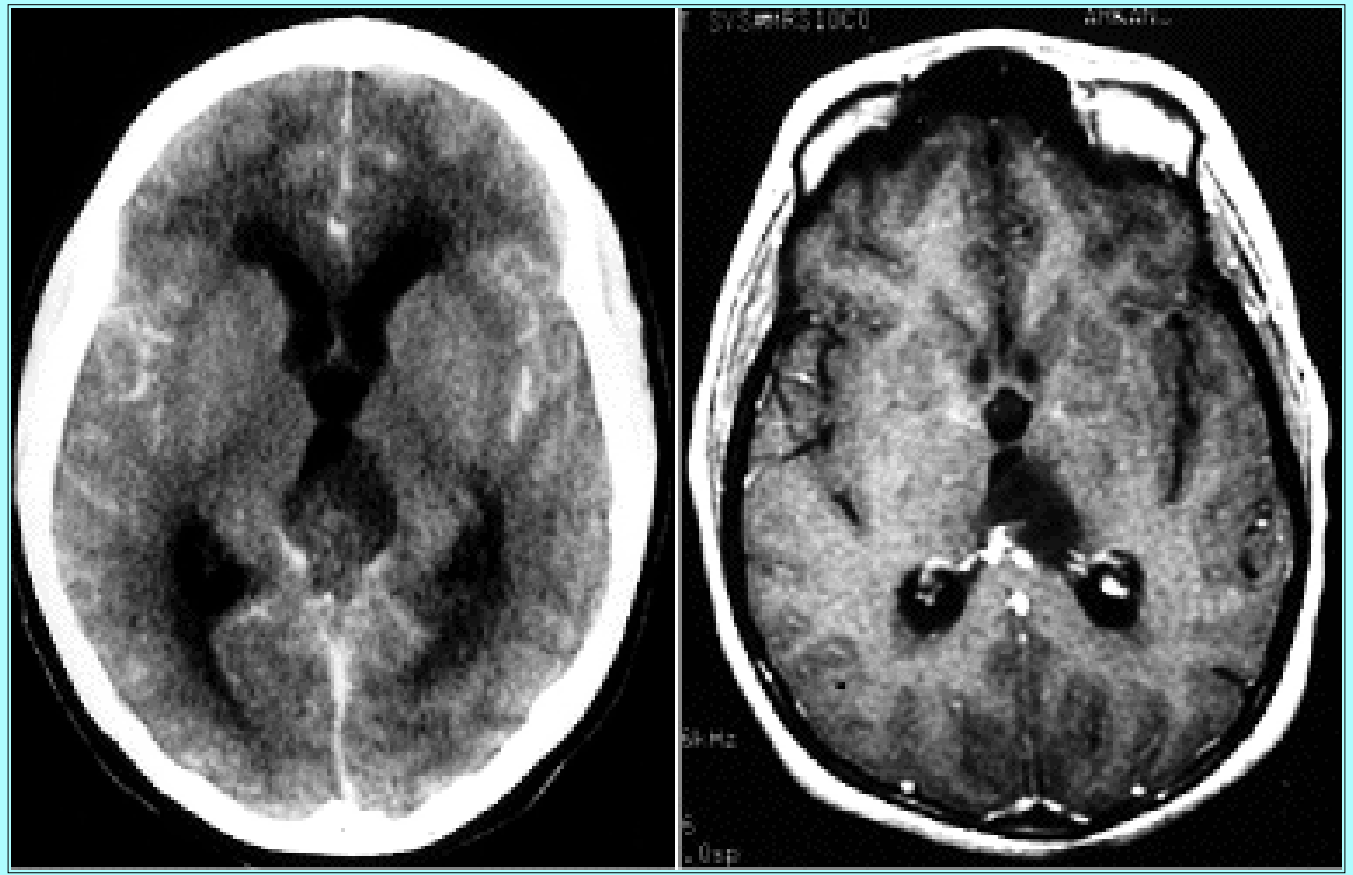

Fig. 1. Case 9. Imaging studies. Left: Preoperative computerized tomography scan revealing hydrocephalus and a posterior third ventricular tumor. This 22-year-old woman was unconscious at presentation and had a history of visual obscurations. She was taken to surgery for emergency ETV. Right: Postoperative magnetic resonance image demonstrating resolution of the hydrocephalus; placement of a shunt and external ventricular drain were not required. 


\section{Technique of Ventriculoscopy, ETV, and Tumor Biopsy}

Patients were given the option of an external ventricular drain or definitive ETV. They were strongly encouraged to choose the endoscopic option if a biopsy sample could be obtained or if the tumor could be treated definitively by endoscopic means (Video Clip 1). They were told that the risk involved with undergoing ETV was slightly higher than that of a simple lateral ventriculostomy but that ETV would provide definitive treatment of their hydrocephalus. They were informed that the risks involved with ETV included death and hypothalamic disturbance as well as infection and other complications associated with any ventriculostomy.

\section{Click here to view Video Clip 1.}

Video Clip 1. This video illustrates two important principles. If the foramen of Monro is not expanded, then the endoscope may be too large to enter the third ventricle. In this situation the "mother-daughter" technique is used. The "mother" scope, a rigid and thicker scope, is first used to define the anatomy and orient the surgeon. The "daughter" scope, a smaller, fiberoptic scope, is then passed into the third ventricle under direct vision. In this particular patient (Case 2), once this was achieved, the pineal cyst was fenestrated, drained, and partially resected.

Once consent was obtained, the patient was taken to the operating room and placed supine on the table with the head flexed at approximately 45 š to the horizontal plane. Placement of the burr hole was determined by preoperative imaging. If the foramen of Monro appeared expanded and the degree of ventricular enlargement was significant, the burr hole was placed approximately halfway between the ideal trajectory for an ETV (1 cm anterior to the coronal suture and $3 \mathrm{cms}$ from the midline) and that for a posterior third ventricular approach. This is usually $3 \mathrm{~cm}$ to the right of the midline and 8 to $10 \mathrm{~cm}$ behind the nasion, which is influenced to some degree by the position of the hairline. After induction of general anesthesia, a 30š angled, rigid endoscope (Ventriculoscope; Aesculap, Tuttlingen, Germany) was placed into the right lateral ventricle. The ventricular wall was inspected for tumor seeding, and a sample of CSF was then taken for cytological examination and analysis of tumor markers. The endoscope was then navigated through the foramen of Monro and the anatomy was defined before any definitive procedure was performed. The technique of third ventriculostomy has been described previously[8-10] and was used in the majority of cases (Fig. 2).

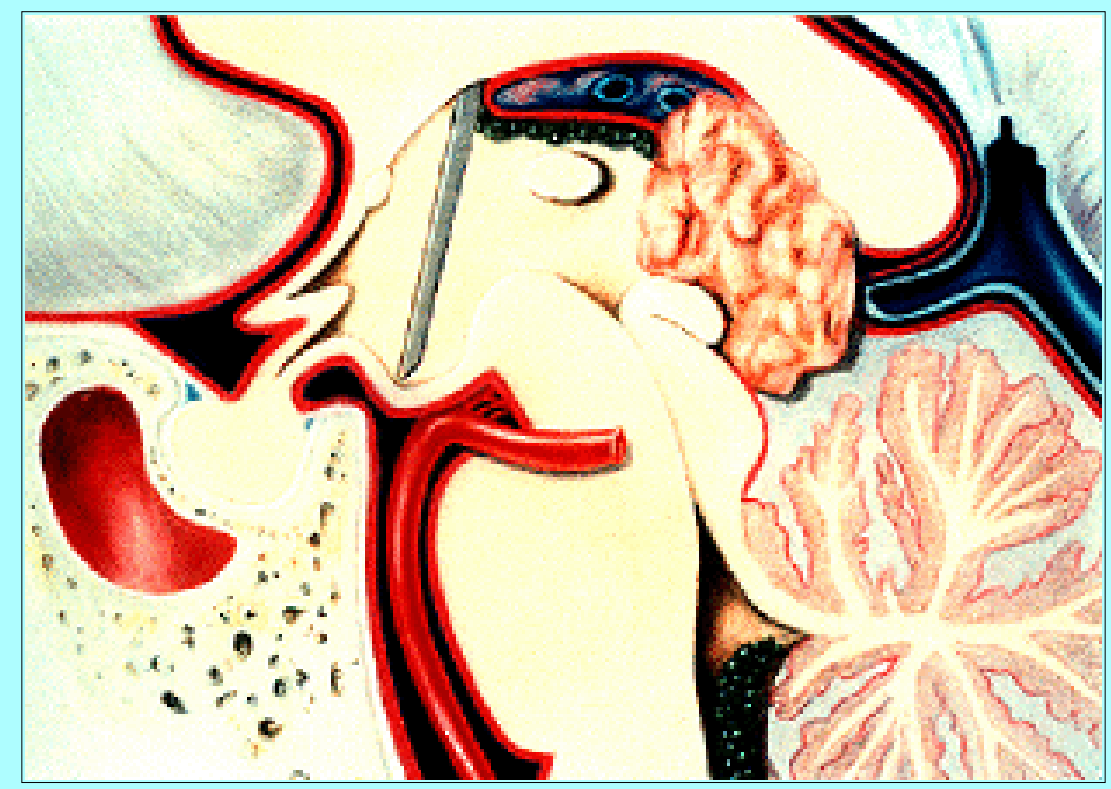


Fig. 2. Drawing of the trajectory for an ETV showing the scope entering the third ventricle through the expanded foramen of Monro and heading toward the anterior part of the floor. The endoscope itself is used to puncture the transparent floor if the basilar complex is visible below.

In those patients with chronic hydrocephalus and large third ventricles, transparently thin floors were demonstrated. However, in some of the patients with very acute onset hydrocephalus their third ventricle floors were thick and opaque. Without the safety of direct visualization of the underlying basilar complex and with a floor that may have been relatively tough, a sharper technique was used to create the stoma (Video Clip 2). The initial hole in the floor was made with the closed end of a pair of grabbing forceps and then expanded using a balloon catheter or the endoscope itself.

\section{Click here to view Video Clip 2.}

Video Clip 2. This is an edited video of the procedures performed in the patient in Case 9 in whom a tumor biopsy sample was obtained and ETV performed. The tumor was thought to be malignant, and hence the ventriculostomy was performed after biopsy sampling. Manipulation of tumor after a communication has been made would theoretically increase the risk of tumor seeding. The rationale for placing the ventriculostomy first is also reasonable. Obtaining a biopsy sample of the tumor may cause bleeding and subsequent clouding of the CSF, making visualization more difficult.

At completion of the ETV, attention was turned to the tumor of the third ventricle. The endoscope was rotated 180 š so that the surgeon had a posterior vantage. A biopsy sample was obtained only if the tumor was visible. In those cases in which the tumor margins were not obvious or if there appeared to be too much functional brain tissue overlying it, the biopsy procedure was aborted. Bleeding at the site at which the biopsy sample was taken invariably stopped with time and moderate irrigation. If the foramen of Monro did not appear to be expanded, if there was only moderate ventriculomegaly, or if the surgeon believed that a biopsy procedure would not alter the future management of the patient, then only ventriculoscopy, CSF sampling and ETV were performed. If the surgeon believed that obtaining a biopsy sample would alter the management algorithm and if the foramen of Monro did not appear to be expanded, then anterior and posterior burr holes were made for access to both the posterior and anterior third ventricle, respectively.

\section{RESULTS}

Of the 19 patients who presented over a 5-year period with posterior third ventricular tumors, 10 were male and nine were female, with an age range of 3 to 28 years (mean age 12 years). Patients were followed for a minimum of 8 months postoperatively (mean of 26 months). In 16 patients hydrocephalus was demonstrated. Five of these patients either had no symptoms or signs of intracranial hypertension or they had longstanding hydrocephalus without acute deterioration, and, therefore, were not offered any form of immediate CSF diversion. In these same patients a craniotomy was performed for the radical total removal of their tumors, and their ventriculomegaly subsequently resolved. Interestingly, one of these patients developed hydrocephalus 3 months after surgery, despite complete removal of a pineal astrocytoma and absence of tumor recurrence. She was treated successfully with ETV.

Of the remaining 11 patients in whom hydrocephalus was demonstrated, six were male and five were female (mean age 11.5 years). All of these patients were managed with endoscopic treatment. This 
consisted of ventriculoscopy, ETV, and in six cases, a biopsy sample of the tumor was obtained. The histopathological classification of their tumors is listed in Table 1. Endoscopy was definitive in four patients. The patient in Case 2 underwent drainage and resection of a posterior third ventricular cyst and did not require a third ventriculostomy (Video Clip 1). The patients in Cases 4, 5, and 6 all harbored presumed tectal gliomas or hamartomas and were treated with definitive ETV in which no biopsy samples were obtained. The patient in Case 6, with neurofibromatosis Type 1, subsequently experienced tumor growth and radiologically evidenced enhancement and therefore underwent a craniotomy for resection of a low-grade astrocytoma.



All patients benefited from endoscopy in some respect. In five patients definitive treatment was achieved with neuroendoscopy, and they required no further surgery. In five patients endoscopic treatment successfully resolved their hydrocephalus. The patient in Case 1 eventually required extracranial CSF 
diversion. However, we believe that this should not be considered a failure because there was a distinct clinical advantage to the delayed placement of a shunt and ETV allowed time for adjuvant therapy to clear the CSF of tumor. In the patient in Case 11 the results of an endoscopically assisted biopsy procedure were proven erroneous 5 days later when radical excision of the tumor demonstrated a malignant nongerminomatous germ cell tumor rather than a benign teratoma. This was more a reflection of the inadequacy of needle or limited biopsy sampling rather than an indictment of endoscopy. There were no endoscopy-related complications in this series.

Although four patients were found to have harbored malignant germ cell tumors, no positive CSF samples were demonstrated. There was significant reduction in ventricular size after ETV in all cases (Fig. 1 right). Of the 16 patients in this series with hydrocephalus, in only two were permanent extracranial shunts implanted. In one patient the shunt was placed at another hospital before referral to the Arkansas Children's Hospital, and in the other patient (Case 1), extensive meningeal seeding of his malignant tumor was demonstrated at the time of surgery. This latter patient underwent surgery and chemotherapy. When recurrence was demonstrated, he underwent radiotherapy followed by high-dose chemotherapy and autologous bone marrow transplantation. He is currently alive and well 6 years after presentation.

\section{DISCUSSION}

The management of hydrocephalus secondary to posterior third ventricular tumors provokes immediate controversy.[6,8] Treatment of all forms of acquired, secondary hydrocephalus depends, to some degree, on the type and the position of the tumor, although the basic principles are similar: avoid shunts if at all possible, treat acute, life-threatening hydrocephalus with immediate CSF diversion, and remove the cause of the obstruction.

The importance of avoiding internalized, extracranial shunts is indisputable. Prolonged extracranial CSF diversion can result in shunt dependency, shunts can become infected and malfunction, and there is a small risk of the tumor spreading into the peritoneal cavity, pleural space, or intravascular compartment, depending on the site in which the distal end is placed. In addition, placement of precraniotomy shunts has been implicated in the genesis of postoperative subdural hematoma due to acute overdrainage. Of course, chronic overdrainage syndromes can occur with shunting for any reason. Externalized CSF diversion offers a reasonable alternative. After the tumor is resected, the drain can be removed with the intention of reestablishing normal CSF pathways, and there is no risk tumor spreading. However, there is the added risk of infection and the need for a second procedure if hydrocephalus persists despite tumor removal or if the tumor recurs at some later date.

Endoscopic third ventriculostomy can be used to address many of these concerns.[1,2,4,11,13] Endoscopic third ventriculostomy is a definitive treatment for all types of noncommunicating hydrocephalus and has well established success rates.[8-10] Successful treatment is even more common in patients with acquired noncommunicating hydrocephalus such as the patients in this series (91\%). The one patient in whom ETV was unsuccessful received considerable benefit despite the eventual failure. Delayed placement of the shunt meant there was no risk of shunt infection while the patient was immunocompromised, there was no added risk of overdrainage in the perioperative period, and the risk of peritoneal metastases was considerably reduced after provision of a course of chemotherapy. Furthermore, ETV offers the added advantage of allowing simultaneous execution of other procedures.[2] The patient in Case 1 did not harbor discernible ventricular metastases on magnetic 
resonance imaging, but an obvious tumor coating the ependymal surface was demonstrated on ventriculoscopy. This was invaluable when staging the tumor resection. Endoscopy allows a biopsy sample of intraventricular tumors to be obtained under direct vision. Although this does not improve the sampling error seen with any form of needle or limited biopsy (as, for example, in Case 11), it does give the surgeon added confidence when acquiring a biopsy sample from more vascularized tumors. Finally, endoscopy may be surgically definitive such as in the patient in Case 2. As illustrated in this case, endoscopic cyst fenestration, drainage, and biopsy sampling may obviate the need for further surgery. With technological advances introduced over the last few years, other intraventricular tumors of the posterior third ventricle such as pedunculated or exophytic gliomas, ependymomas, and posteriorly placed colloid cysts can be completely removed endoscopically.[7,14]

The obvious downside to ETV is its added risk to the patient[12] over a simple external ventricular drain. Although the complication rate in this series was $0 \%$, there have been several reports in the recent literature that underscore the dangers of imprecise puncture of the third ventricular floor.[3,5] The authors concede that ETV is not without risk but strongly believe the risk is considerably less if the floor is punctured when using the blunt technique, as described by the primary author in previous publications.[8-10] The blunt technique cannot be used in all cases. In these situations the following algorithm may be useful. Patients with symptomatic hydrocephalus who present acutely should be considered for ventriculoscopy as well as either external ventricular drainage or third ventriculostomy. Using a right frontal burr hole, the lateral ventricle should be entered and the third ventricle inspected. If the floor is not transparently thin and cannot be punctured safely, a drain is left in the lateral ventricle after inspection, and CSF and biopsy sampling have been performed. A cautious approach to ETV will secure a permanent place for endoscopy in the management of these patients.

In conclusion, endoscopy can play a major role in the management of patients with tumors of the posterior third ventricle. The results of this series demonstrate the safety and efficacy of this technique with a $0 \%$ complication rate, and good or excellent outcome was obtained in $91 \%$ of cases after undergoing the various endoscopic procedures. It may offer definitive treatment for secondary hydrocephalus as well as the primary condition, and it can be an excellent tool in tumor staging and diagnosis. Although endoscopy is not without risks, these can be reduced with experience and judicious selection of patients.

\section{References}

1. Ellenbogen RG, Moores LE: Endoscopic management of a pineal and suprasellar germinoma with associated hydrocephalus: technical case report. Minim Invasive Neurosurg 40:13-15, 1997

2. Ferrer E, Santamarta D, Garcia-Fructuoso G, et al: Neuroendoscopic management of pineal region tumours. Acta Neurochir 139:12-20, 1997

3. Gaab MR, Schroeder HWS: Neuroendoscopic approach to intraventricular lesions. J Neurosurg 88:496-505, 1998

4. Handler MH, Abbott R, Lee M: A near-fatal complication of endoscopic third ventriculostomy: case report. Neurosurgery 35:525-528, 1994

5. Robinson S, Cohen AR: The role of neuroendoscopy in the management of pineal region tumors. Surg Neurol 48:360-367, 1997 
6. Schroeder HWS, Warzok RW, Assaf JA, et al: Fatal subarachnoid hemorrhage after endoscopic third ventriculostomy. J Neurosurg 90:153-155, 1999

7. Stein BM, Bruce JN: Surgical management of pineal region tumors. Clin Neurosurg 39:509-532, 1992

8. Teo C: Complete endoscopic removal of colloid cysts: issues of safety and efficacy. Neurosurg Focus 6 (4): Article 9, 1999

9. Teo C: Endoscopy for the treatment of hydrocephalus, in King WA, Frazee JG, DeSalles AAF (eds): Endoscopy of the Central and Peripheral Nervous System. New York: Thieme, 1998, pp 59-67

10. Teo C: Third ventriculostomy in the treatment of hydrocephalus: experience with more than 120 cases, in Hellwig D, Bauer BL (eds): Minimally Invasive Techniques for Neurosurgery: Current Status and Future Perspectives. Berlin: Springer-Verlag, 1998, pp 73-76

11. Teo C, Jones RF: Management of hydrocephalus by endoscopic third ventriculostomy in patients with myelomeningocele. Pediatr Neurosurg 25:57-63, 1996

12. Teo C, Rahman S, Boop FA, et al: Complications of endoscopic neurosurgery. Childs Nerv Syst 12:248-253, 1996

13. Turtz AR, Hughes WB, Goldman HW: Endoscopic treatment of a symptomatic pineal cyst: technical case report. Neurosurgery 37:1013-1015, 1995

14. Veto F, Horvath Z, Doczi T: Biportal endoscopic management of third ventricle tumors in patients with occlusive hydrocephalus: technical note. Neurosurgery 40:871-877, 1997

Manuscript received August 19, 1999.

Accepted in final form September 21, 1999.

Address reprint requests to: Charles Teo M.B.B.S., F.R.A.C.S., Centre for Minimally Invasive Neurosurgery, Prince of Wales Hospital/University of New South Wales, Suite 3, Level 7, POW Private Hospital, Barker Street, Randwick, Sydney, New South Wales, 2031, Australia. 